\title{
Magnetic Behaviour of Core/Shell Nanoparticle Assemblies: Interparticle Interactions Effects
}

\author{
K. Trohidou* AND M. VASILAKAKI \\ Computational Materials Science Group \\ Institute of Materials Science, NCSR Demokritos, 15310 Athens, Greece
}

\begin{abstract}
Interparticle interactions in assemblies of nanoparticles represent an important effect that modifies their magnetic behavior. The characteristics of the hysteresis loop and the temperature dependent magnetization (field cooled (FC)/zero-field cooled (ZFC)) are studied numerically in magnetic nanoparticle assemblies using Monte Carlo simulations and the standard Metropolis algorithm. We study composite spin nanostructures with ferromagnetic (FM) core/antiferromagnetic (AFM) shell morphology and simple ferromagnetic nanostructures. Both three-dimensional granular solids and two-dimensional ordered nanoparticle arrays are analyzed and compared, with emphasis on the interplay between single-particle characteristics and magnetostatic interparticle interactions. We find that the antiferromagnetic interface provides a strong exchange coupling with the ferromagnetic core and, through it, an effective long range interparticle correlation, modified from that of simple FM nanostructures. The results of our simulations are compared with experimental findings.
\end{abstract} 75.75. $+\mathrm{a}$

PACS numbers: 75.50.Tt, 75.40.Mg, 75.50.Bb, 75.50.Vv, 75.30.Et, 75.60.Ej, 75.50.Lk, 75.50.Cc, 75.50.Ss,

\section{Introduction}

Two distinct growth techniques, namely, the cluster beam deposition [1] of preformed nanoparticles and the self-assembly [2-4] of nanoparticles prepared in a colloidal dispersion, produce nanostructured films composed of magnetic nanoparticles with an extremely narrow size distribution. The nature of the interparticle interactions and the film morphology are different in these two cases. With the former method, random assemblies of nanoparticles are formed and strong interparticle exchange interactions are expected to occur when nanoparticles are in contact [5]. These interactions become the dominant ones at concentrations close to the percolation threshold. In the latter case, nearly planar hexagonal arrays are formed and dipolar interparticle interactions dominate [6]. Exchange interactions are suppressed in this case by the presence of a thick surfactant layer.

In the continuous research effort for development of magnetic nanostructures with reduced size and improved thermal stability [7], composite nanoparticles with a FM core and an AFM shell have attracted a lot of interest [8]. Atomic scale models of the magnetic structure and the exchange bias effect in these composite nanoparticles, have supported experimental observations initially in transition metal nanoparticles with an oxidized shell.

Among the most important theoretical results [9] we mention (i) the disappearance of the exchange bias field

* corresponding author; e-mail: trohidou@ims.demokritos.gr
$\left(H_{\mathrm{E}}\right)$ at temperatures above the Néel temperature of the AFM, in agreement with experiments [10], (ii) the strong dependence of the exchange field $H_{\mathrm{E}}$ of the number uncompensated bonds across the FM-AFM interface and the dependence of the coercive field $H_{\mathrm{C}}$ on the interface area, (iii) the increase in both $H_{\mathrm{E}}$ and $H_{\mathrm{C}}$ for a given nanoparticle radius with increasing shell thickness, (iv) the increase in $H_{\mathrm{E}}$ and decrease in $H_{\mathrm{C}}$ with increasing oxidation layer thickness and a fixed core radius, (v) the fast stabilization of $H_{\mathrm{E}}$ with increasing core size, in agreement with experiments [11], and (vi) the reduction in $H_{\mathrm{C}}$ and increase in $H_{\mathrm{E}}$ and its thermal stability with increasing exchange constant of the AFM material and/or at the FM-AFM interface. Despite the research effort focused on the microscopic mechanism of the exchange bias effect in individual nanoparticles [9, 12], much less attention has been paid so far to the modification of the magnetic hysteresis behaviour due to inter-particle interactions arising in assemblies. In this direction, Fe nanoparticles embedded in iron-oxide matrix [13] were shown to freeze below a temperature owing to the competition between the exchange anisotropy at the core-shell interface and the interparticle dipole-dipole interactions. Similarly, increase in the exchange bias field due to magnetostatic interparticle coupling was found in stripes of $\mathrm{Co} / \mathrm{CoO}$ nanoparticles [14] and inter-dot magnetostatic interactions were shown to produce asymmetric anomalies in the magnetization reversal mechanism of $\mathrm{Co} / \mathrm{CoO}$ dot arrays [15]. The modification of the coercive and exchange bias fields in dense nanoparticle arrays with core-shell morphology as a result of the competition be- 
tween exchange anisotropy and interparticle dipolar interactions presents a challenging issue.

In this work we implement the Monte Carlo simulation method to investigate the magnetic properties of these two categories of core/shell nanoparticle assembled systems. The purpose of our work is the study of the importance of interparticle interactions in core/shell nanoparticle systems and the comparison of their magnetic behaviour with that of single spin Stoner Wohlfarth (SW) type nanoparticle assemblies.

The paper is organised as follows: in section 2 we describe the model of the magnetic structure of nanoparticles and the method of calculation of the exchange bias field and the coercive field in 3-D random assemblies of nanoparticles, and 2-D ordered arrays; in section 3 we present numerical results and discussion for the magnetic behaviour of two structures of assemblies and within each kind of structure, the differences in behaviour of composite nanoparticles and single spin nanoparticles.

\section{Model and simulation method}

Two different models for spatial arrangements of the particle assembly are considered. In the first model, spherical particles are located randomly with occupation probability $p$ inside a cubic box of edge length $L$. The particles are located only at lattice sites, thus overlap is avoided. This geometrical model describes the morphology of films formed by co-deposition of preformed particles with non-magnetic atoms [16]. In the second model, the particles form a two-dimensional triangular lattice in the $x y$-plane with lattice constant $d_{0}$. This model describes adequately the morphology of self-assembled ordered arrays of magnetic nanoparticles [17, 18].

Initially we consider the magnetic state of the single spin nanoparticles with particle diameter $D$, which is described by the Stoner-Wohlfarth model that assumes coherent rotation of the particle's magnetization. An anisotropy axis in a random direction is attributed to each particle. The particles interact via long range dipolar forces and via exchange forces, when they are sufficiently close. The particle assembly is assumed monodisperse in accordance with experimental evidence that both films grown by cluster beam [16] or by self-assembly on surfaces $[17,18]$ are characterized by extremely low size dispersion. The total energy of the nanoparticles' assembly is

$$
\begin{aligned}
E & =g \sum_{i, j} \frac{\left(\hat{\boldsymbol{S}}_{i} \cdot \hat{\boldsymbol{S}}_{j}\right)-3\left(\hat{\boldsymbol{S}}_{i} \cdot \hat{\boldsymbol{R}}_{i j}\right)\left(\hat{\boldsymbol{S}}_{j} \cdot \hat{\boldsymbol{R}}_{i j}\right)}{R_{i j}^{3}} \\
& -J \sum_{\langle i, j\rangle}\left(\hat{\boldsymbol{S}}_{i} \cdot \hat{\boldsymbol{S}}_{j}\right)-k \sum_{i}\left(\hat{\boldsymbol{S}}_{i} \cdot \hat{\boldsymbol{e}}_{i}\right)^{2} \\
& -h \sum_{i}\left(\hat{\boldsymbol{S}}_{i} \cdot \hat{\boldsymbol{H}}\right)
\end{aligned}
$$

where $\hat{\boldsymbol{S}}_{i}$ is the magnetic moment direction (spin) of particle i, $\hat{\boldsymbol{e}}_{i}$ is the easy axis direction, $\hat{\boldsymbol{R}}_{i j}$ is the center-to-center distance between particles $\mathrm{i}$ and $\mathrm{j}$, measured in units of the particle diameter. The energy parameters entering Eq. (1) are the dipolar energy with dipolar coupling constant $g$, the exchange energy with exchange coupling constant $\mathrm{J}$, the anisotropy energy with anisotropy constant $\mathrm{k}$ and the Zeeman energy $h=\mu H$, where $\mu=M_{\mathrm{s}} V_{\mathrm{o}}$ is the particle magnetic moment. The exchange coupling decays within a few lattice constants of the parent magnetic material, therefore, it is restricted in our model to the particles in contact (nearest neighbours) only. Consequently, it does not occur in the model that describes the ordered arrays, which are not in contact.

For assemblies of composite nanoparticles we consider identical spherical particles with core diameter $D$ and shell diameter $D_{0}$. In each nanoparticle, the FM core and the interface have uniaxial anisotropy and the AFM shell has random anisotropy and much higher than the core anisotropy. Under these assumptions the total energy of the assembly is given as

$$
\begin{aligned}
E & =g \sum_{i, j} \frac{\left(\hat{\boldsymbol{S}}_{i} \cdot \hat{\boldsymbol{S}}_{j}\right)-3\left(\hat{\boldsymbol{S}}_{i} \cdot \hat{\boldsymbol{R}}_{i j}\right)\left(\hat{\boldsymbol{S}}_{j} \cdot \hat{\boldsymbol{R}}_{i j}\right)}{R_{i j}^{3}} \\
& -J \sum_{\langle i, j\rangle}\left(\hat{\boldsymbol{S}}_{i} \cdot \hat{\boldsymbol{S}}_{j}\right)-K_{\mathrm{C}} \sum_{i}\left(\hat{\boldsymbol{S}}_{i} \cdot \hat{\boldsymbol{e}}_{i}\right)^{2} \\
& -K_{\mathrm{SH}} \sum_{i}\left(\hat{\boldsymbol{S}}_{i} \cdot \hat{\boldsymbol{e}}_{i}\right)^{2}-h \sum_{i}\left(\hat{\boldsymbol{S}}_{i} \cdot \hat{\boldsymbol{H}}\right),
\end{aligned}
$$

where $K_{\mathrm{C}}, K_{\mathrm{SH}}$ are the anisotropy constants and $J$ is the exchange coupling constant across the core-shell interface of the nanoparticle. Long-range dipolar interactions between the cores are included with dipolar coupling constant $g$ and the Zeeman energy $\mathrm{h}=\mu \mathrm{H}$, where $\mu=M_{\mathrm{s}} V_{\mathrm{o}}$ is the particle magnetic moment, as they appear in Eq. (1). The exchange field $\left(H_{\mathrm{E}}\right)$ and the effective coercive field $\left(H_{\mathrm{C}}\right)$ are given as $H_{\mathrm{E}}=\left|H_{\mathrm{C} 2}+\mathrm{H}_{\mathrm{C} 1}\right| / 2$ and $H_{\mathrm{C}}=\left|H_{\mathrm{C} 2}-\mathrm{H}_{\mathrm{C} 1}\right| / 2$, where $H_{\mathrm{C} 1}$ and $H_{\mathrm{C} 2}$ are the left and right coercive fields, respectively.

Comparing Eq. (1) with Eq. (2), we see that the main difference comes from the fact that in Eq. 2 the intra-particle characteristics of each composite nanoparticle, namely, the core, the shell anisotropy energy and mainly the exchange interaction between the core and the shell, are entering in the total energy.

In the model that describes the granular solids, periodic boundaries in all directions are used, while in the second model for the ordered arrays mixed periodic boundaries ( $x y$-plane) and open boundaries (z-axis) are used. Dipolar forces are summed to be on an infinite range, using Ewald's method when the boundaries are periodic. The spin configuration is obtained by a Metropolis Monte Carlo algorithm [19]. In all loops presented below the magnetization is reduced to the total volume. At a given temperature and applied field, the system is allowed to relax towards equilibrium for the first $10^{2}$ Monte Carlo steps per spin and thermal averages are calculated over the subsequent $10^{4}$ steps. The results are averaged over 10-30 samples with different particle configurations. 


\section{Results and discussion}

In what follows we present our numerical results for granular nanoparticle assemblies and for ordered arrays. Discussion for the comparison between the single nanoparticle and composite nanoparticle assemblies will be given in both models.

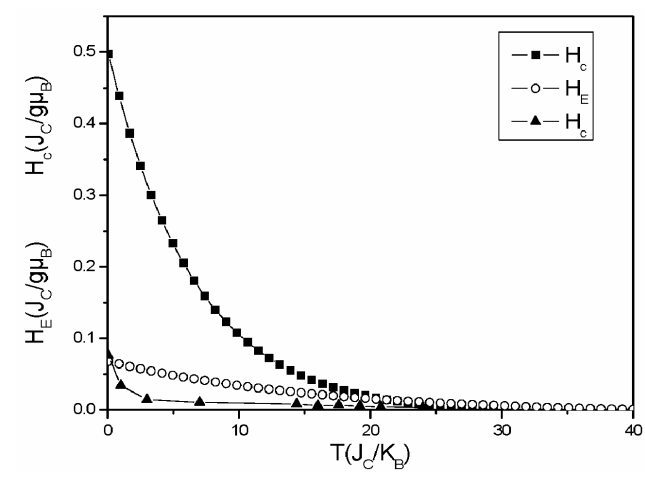

Fig. 1. Temperature dependence of $H_{\mathrm{C}}$ and $H_{\mathrm{E}}$ for the random assembly of composite nanoparticles, and $H_{\mathrm{C}}$ for the single spin nanoparticle assembly.

We first start with the magnetic behaviour of granular systems. The hysteresis loops of a random assembly of composite nanoparticles with core/shell morphology at low concentration has been calculated at different temperatures. Also, we have calculated the hysteresis loops of a random assembly of single spin nanoparticles at the same low concentration, for the same range of temperatures. The results are given in Fig. 1 where we plotted the temperature dependence of $H_{\mathrm{E}}$ (open circles) and $H_{\mathrm{C}}$ (squares) for the random assembly of composite nanoparticles together with $H_{\mathrm{C}}$ (triangles) of the single spin assembly. As we can see apart from the fact that $H_{\mathrm{C}}$ is lower for the single spin assembly for the whole range of temperatures, in the case of composite nanoparticles it remains finite at higher temperatures. This is due to the extra anisotropy of composite nanoparticles, induced by the exchange coupling along the interface $[8,9,20]$. Also, in composite nanoparticles we observe an exponential decay of $H_{\mathrm{C}}$ and $H_{\mathrm{E}}$ with the temperature.

In Fig. 2 we plot the ZFC magnetization curves for composite nanoparticle assemblies (squares) and for single spin nanoparticle assemblies (triangles). The maximum of the ZFC is considered as the blocking temperature $T_{\mathrm{b}}$ of the system. We observe that the blocking temperature in the first case is higher than in the second, in agreement with experimental findings [20]. Namely, the blocking temperature increases in the case of the composite nanoparticles due to the extra exchange anisotropy along the interface.

Next, we examine the magnetic behaviour of the ordered arrays of nanoparticles. We start with the study of the magnetic behaviour of single spin nanoparticle ordered arrays. In Fig. 3a we plotted the ZFC curves for various interparticle distances $\left(d_{0}\right)$ ranging from the

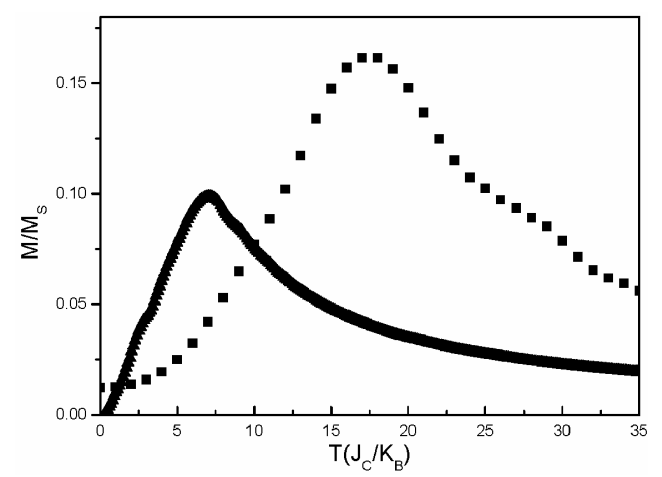

Fig. 2. Zero-field cooled curves for the random assembly of composite nanoparticles (squares) and for the single spin nanoparticle assembly (triangles).
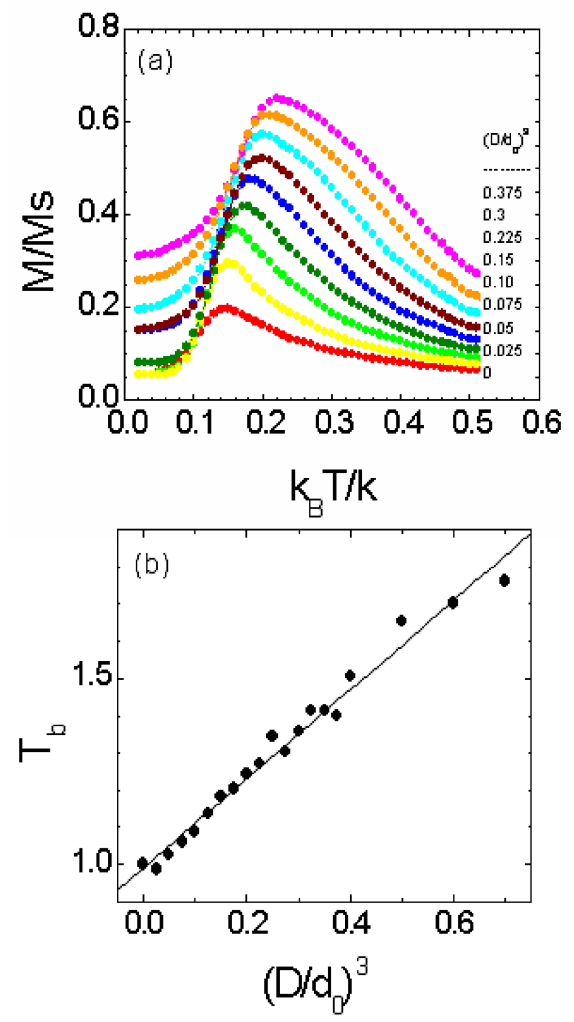

Fig. 3. (a) Dependence of zero-field cooled magnetization on interparticle distance $\left(\mathrm{D} / \mathrm{d}_{0}\right)^{3}$. (b) The blocking temperature $\left(T_{\mathrm{b}}\right)$ as a function of the inverse cube of the nanoparticle distance $\left(\mathrm{D} / \mathrm{d}_{0}\right)^{3}$.

closest case $\left(D^{3} / d_{0}^{3}=0.375\right)$ to infinite separation $\left(D^{3} / d_{0}^{3}=0\right)$. The dipolar interactions, as nanoparticles come closer, provide a stronger additional energy barrier for the magnetization reversal enhancing the magnetic stability of the system and as a result we have a higher blocking temperature. Our data (Fig. 3b) indicate also that the blocking temperature scales with the inverse cube of the nanoparticle distance $\left(T_{\mathrm{b}} \sim 1 / d_{0}^{3}\right)[21$. 


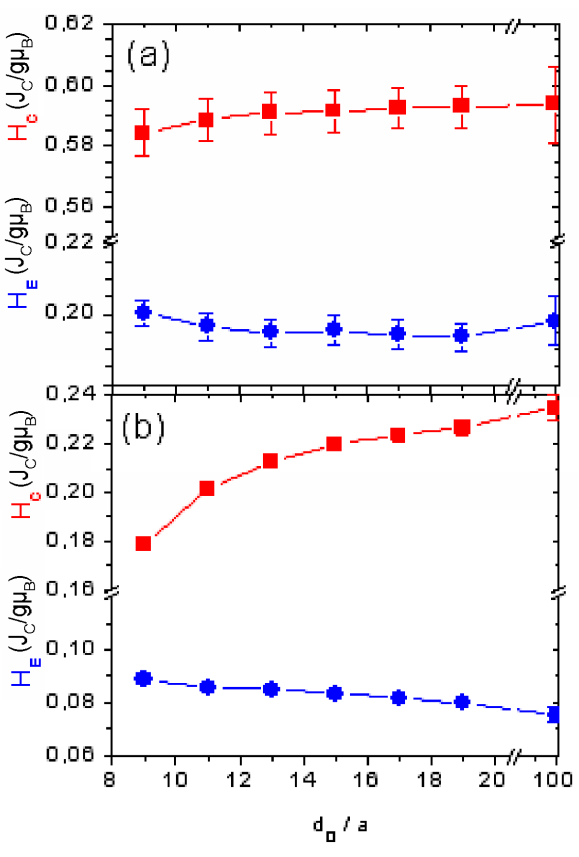

Fig. 4. Dependence of $H_{\mathrm{C}}$ and $H_{\mathrm{E}}$ on the interparticle spacing $\left(d_{0}\right)$ in 2-D hexagonal array of nanoparticles with core radius $5 a$ and external radius $9 a$ for: (a) weakly dipolar $(g=1.0)$ material and (b) for strongly dipolar $(g=6.5)$ material.

We examined also the behaviour of the coercive and the exchange bias fields in the presence of dipolar interparticle interactions in two-dimensional hexagonal ordered arrays of composite nanoparticles with FM core radius $5 a$ and AFM shell radius $4 a$, where $a$ is the lattice constant. The effect of the interparticle distance $\left(d_{0}\right)$ was examined for two different values of the dipolar coupling constant $g$, which depends on the material. In Figs. $4 \mathrm{a}-\mathrm{b}$ we plot the coercive field and the exchange bias field for various interparticle distances $\left(d_{0}\right)$, ranging from $\left(d_{0} / a=9\right)$ to a large separation $\left(d_{0} / a=100\right)$. We show results for ordered nanoparticle arrays with a weak $(g=1$, Fig. 4a) and strong ( $g=6.5$, Fig. $4 \mathrm{~b})$ dipolar coupling strength. We observe that the reduction of the interparticle distance causes the reduction of the coercivity due to collective response of magnetic moments, that leads to a reduction of the energy barrier for magnetization reversal. As shown in Fig. 4, the reduction of the $H_{\mathrm{C}}$ is more pronounced in strongly dipolar materials. The decrease of the interparticle distance enhances the collective rotation of magnetic moments in a strongly dipolar system.

With increasing the interparticle distance, the exchange bias field is less affected (Figs. 4a-b). For weak dipolar materials $H_{\mathrm{E}}$ is slightly increased with the decrease in $d_{0}$ though for stronger ones it clearly increases. The dipole-dipole interactions enhance the exchange bias effect. We have found a similar increase in $H_{\mathrm{E}}$ with dipolar coupling strength in Ref. [22]. We attribute this behaviour to the competition between exchange anisotropy and dipolar interactions.

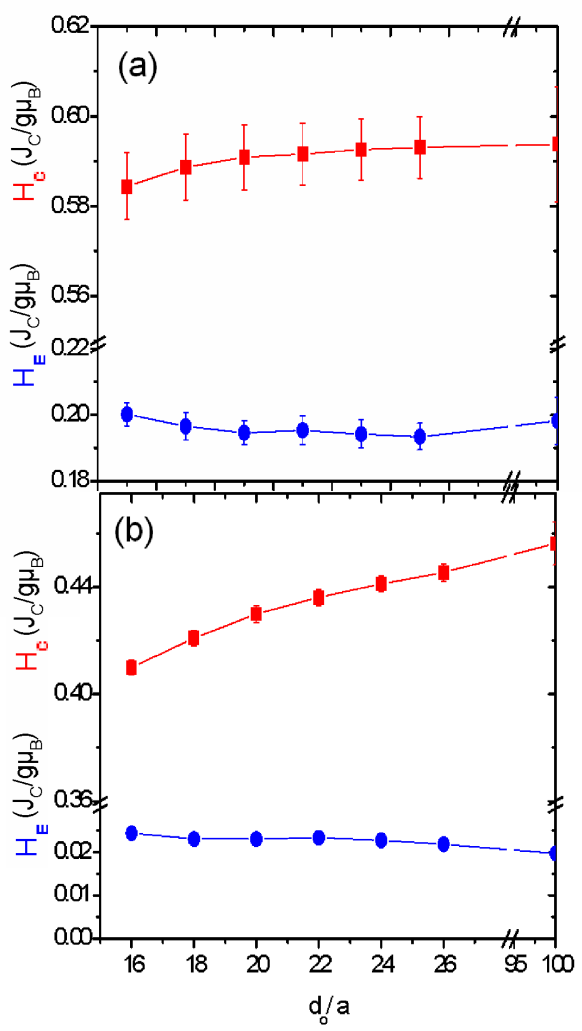

Fig. 5. Dependence of $H_{\mathrm{C}}$ and $H_{\mathrm{E}}$ on the interparticle spacing $\left(d_{0}\right)$ in 2-D hexagonal array of nanoparticles with shell thickness $4 a$ and the core radius (a) $5 a$ and

(b) $12 a$, for a weakly dipolar $(g=1.0)$ material.

In order to understand better the role of the core to the magnetic behaviour of ordered arrays of composite nanoparticles, we have investigated the effect of the core radius on $H_{\mathrm{E}}$ and $H_{\mathrm{C}}$. In Figs. $5 \mathrm{a}-\mathrm{b}$ we plot the coercivity and exchange bias field for two different core radii $5 a$ and $12 a$, respectively. We observe that the increase in $H_{\mathrm{C}}$ with the increase in the interparticle distance becomes stronger for the bigger nanoparticle due to its higher anisotropy energy. $H_{\mathrm{E}}$ increases very little with the decrease in $d_{0}$. The values of $H_{\mathrm{E}}$ and $H_{\mathrm{C}}$ are smaller in the case of bigger core diameter in agreement with our previous results for atomic scale models [9], where structural and magnetic details at the interface are taken into account explicitly.

\section{Conclusions}

The role of dipolar interparticle interactions in the magnetic behaviour of assemblies of single spin nanoparticles and composite FM core/AFM shell nanoparticles has been investigated by the Monte Carlo simulations method.

The random assemblies of composite FM core/AFM shell nanoparticles exhibit: a) higher $H_{\mathrm{C}}$ than the SW 
type nanoparticle assemblies and the appearance of $H_{\mathrm{E}}$, b) exponential decay of $H_{\mathrm{E}}$ and $H_{\mathrm{C}}$ with the temperature, c) higher $T_{\mathrm{b}}$ than the SW type nanoparticle assemblies, in agreement with the $\mathrm{Co} / \mathrm{Mn}$ experiments $[17,20]$.

The ordered arrays of single spin nanoparticle assemblies exhibit a decay of $T_{\mathrm{b}}$ with the cube of interparticle distance. The dipolar interparticle interactions in ordered arrays of FM core/AFM shell nanoparticles facilitate the magnetization reversal in both directions. Therefore, $H_{\mathrm{C}}$ increases with the interparticle distance and $H_{\mathrm{E}}$ increases slightly. The increase of $H_{\mathrm{C}}$ is more pronounced in strongly dipolar materials and for large nanoparticles.

\section{References}

[1] A. Perez, P. Melinon, V. Dupuis, P. Jensen, B. Prevel, J. Tuaillon, L. Bardotti, C. Martet, M. Treilleux, M. Broyer, M. Pellarin, J.L. Vaille, B. Palpant, J. Lerme, J. Phys. D, Appl. Phys. 30, 709 (1997).

[2] S. Sun, C.B. Murray, J. Appl. Phys. 85, 4325 (1999).

[3] V.F. Puntes, K.M. Krishnam, A.P. Alivisatos, Appl. Phys. Lett. 78, 2187 (2001).

[4] V. Russier, C. Petit, J. Legrand, M.P. Pileni, Phys. Rev. B 62, 3910 (2000).

[5] J. Nogues, V. Skumryev, J. Sort, S. Stoyanov, D. Givord, Phys. Rev. Lett. 97, 157203 (2006).

[6] I. Lisiecki, M. Walls, D. Parker, M.P. Pileni, Langmuir 24, 4295 (2008).

[7] V. Skumryev, S. Stoyanov, Y. Zhang, G. Hadjipanayis, D. Givord, J. Nogues, Nature (London) 423 850 (2003).

[8] J. Nogues, J. Sort, V. Langlais, V. Skumryev, S. Surinach, J.S. Munoz, M.D. Baro, Phys. Rep. 422, 65 (2005).
[9] E. Eftaxias, K.N. Trohidou, Phys. Rev. B 71, 134406 (2005).

[10] J. van Lierop, M.A. Schofield, L.H. Lewis, R.J. Gambino, J. Magn. Magn. Mater. 264, 146 (2003).

[11] S. Gangopadhyay, G.C. Hadjipanayis, C.M. Sorensen, K.J. Klabunde, J. Appl. Phys. 73, 6964 (1993).

[12] M. Vasilakaki, E. Eftaxias, K.N. Trohidou, Phys. Stat. Sol. A 205, 1865 (2008).

[13] L. Del, D. Bianco, A.M. Fiorani, E. Testa, L. Bonetti, S. Savini, Signoretti, Phys. Rev. B 66, 174418 (2002).

[14] H. Bi, S. Li, X. Jiang, Y. Du, C. Yang, Phys. Lett. A 307, 69 (2003).

[15] K. Temst, E. Popova, H. Loosvelt, M.J. Van, S. Bael, Y. Brems, C. Bruynseraede, Van Haesendonck, H. Fritzcheb, M. Gierlings, L.H.A. Leunissen, R. Jonckheere, J. Magn. Magn. Mater. 304, 14 (2006).

[16] C. Binns, M.J. Maher, Q.A. Pankhurst, D. Kechrakos, K.N. Trohidou, Phys. Rev. B 66, 184413 (2002).

[17] N. Domingo, D. Fiorani, A.M. Testa, C. Binns, S. Baker, J. Tejada, J. Phys. D, Appl. Phys. 41, 134009 (2008).

[18] C.B. Murray, S. Sun, H. Duyle, T. Betley, MRS Bull. 26, 985 (2001).

[19] K. Binder, D.W. Heermann, Monte Carlo Simulation in Statistical Physics, Springer Series in Solid-State Sciences, 80, Springer-Verlag, Berlin 1998.

[20] N. Domingo, A.M. Testa, D. Fiorani, C. Binns, S. Baker, J. Tehada, J. Magn. Magn. Mater. 316, 155 (2007).

[21] D. Kechrakos, K.N. Trohidou, Appl. Phys. Lett. 81, 4574 (2002).

[22] D. Kechrakos, K.N. Trohidou, M. Vasilakaki, J. Magn. Magn. Mater. 316, e291 (2007). 